\title{
Three-Dimensional Remnant Pancreatic Volumetry Predicts Postoperative Pancreatic Fistula in Pancreatic Cancer Patients after Pancreaticoduodenectomy
}

\author{
Ryoichi Miyamoto $^{a}$ b Yukio Oshiro $^{b}$ Naoki Sano $^{a}$ Satoshi Inagawa ${ }^{a}$ \\ Nobuhiro Ohkohchi ${ }^{\text {b }}$ \\ a Department of Gastroenterological Surgery, Tsukuba Medical Center Hospital, \\ Tsukuba, Japan; ${ }^{b}$ Division of Gastroenterological and Hepatobiliary Surgery and Organ \\ Transplantation, Department of Surgery, University of Tsukuba, Tsukuba, Japan
}

\author{
Keywords \\ Pancreatic cancer - Volumetry · Pancreatic fistula - Postoperative pancreatic fistula . \\ Pancreaticoduodenectomy
}

\begin{abstract}
Background: Postoperative pancreatic fistula (POPF) is a serious complication that can occur following pancreaticoduodenectomy (PD). Recent studies suggest that remnant pancreatic volume (RPV) values from preoperative multidetector computed tomography (MDCT) are highly predictive of POPF. We performed three-dimensional (3D) surgical simulation of PD including RPV measurements. The aim of this study was to determine whether 3D-measured RPV is predictive of POPF after PD. Methods: We used the SYNAPSE VINCENT ${ }^{\circledR}$ medical imaging system (Fujifilm Medical Co., Ltd., Tokyo, Japan) to construct 3D images after integrating MDCT and magnetic resonance cholangiopancreatography images. RPV was measured using this 3D image, which simulated actual intraoperative pancreatic parenchymal remnant volume. Ninety-one patients who underwent PD were retrospectively enrolled. Using multivariate analysis, RPV and other well-known POPF risk factors were independently assessed. $\boldsymbol{R e}$ sults: Multivariate analysis identified high RPV values (hazard ratio $[H R]=8.41, p=0.01$ ), pancreatic duct diameter $<3.0 \mathrm{~mm}(\mathrm{HR}=5.48, p<0.01)$, no pathological fibrosis $(\mathrm{HR}=3.41$, $p<0.01)$, and body mass index $>25 \mathrm{~kg} / \mathrm{m}^{2}(\mathrm{HR}=1.53, p=0.02)$ as independent risk factors for POPF. Conclusion: The present study indicates that preoperative 3D-measured RPV is predictive of POPF after PD.




\section{Introduction}

Pancreaticoduodenectomy (PD) remains the foundation of curative surgical treatments for pancreatic head cancer. Recent improvements in operative techniques and perioperative management have substantially decreased postoperative mortality rates to $<5 \%[1,2]$. Nevertheless, the postoperative morbidity rate remains high (30-50\%), even in high-volume centers $[3,4]$. Postoperative pancreatic fistula (POPF) is the most common major complication of PD, with a frequency ranging from 2 to $20 \%$ [3, 4]. POPF can lead to abscess formation, vascular injuries, pseudoaneurysm rupture, fatal hemorrhage, and sepsis. Therefore, reducing the rate of POPF has been an important but challenging goal for pancreatic surgeons.

Several risk factors for the development of clinically relevant POPF have been defined and validated, and these factors can be divided into pancreas-related factors, such as a soft pancreas, pancreatic pathology, and small main pancreatic duct size $(\leq 3.0 \mathrm{~mm})$; patient-related factors, such as advanced age, male sex, and high body mass index (BMI); and surgery-related factors, such as long operation times, significant blood loss, pancreaticojejunal anastomotic stents, and pancreaticojejunostomy procedures, including the Blumgart method or the Kakita method [5-10]. However, the clinical usefulness of these factors is limited by the fact that they are determined through subjective intraoperative examination. Ideally, predictive factors should be determined preoperatively by objective parameters. Preoperative identification of patients at high risk of clinically relevant POPF can increase the accuracy of preoperative benefit/risk assessment and information given to patients and should enable surgeons to modify surgical procedures and perioperative management to reduce risk [11-13].

Recent advances in workstations for radiological diagnostic imaging have made it easier to construct three-dimensional (3D) images of vasculature and organs. In particular, 3Dmeasured volumetry is widely used for hepatic resection to facilitate risk prediction as early as the preoperative setting [14-16]. A recent study suggested that a large remnant pancreatic volume (RPV), as estimated using preoperative multidetector computed tomography (MDCT) images, significantly and independently affected the risk of POPF $[13,17,18]$. However, RPV estimated from MDCT was insufficient to simulate the actual pancreatic dissection position and precisely measure RPV. Therefore, we developed a novel method for combining MDCT and magnetic resonance cholangiopancreatography (MRCP) images to perform 3D surgical simulations of pancreatic surgery [19-22]. By integrating these two images, we can visualize the anatomic relationships between nearby vascular structures and the pancreas. Furthermore, we are able to simulate the pancreatic dissection line using anatomic images before the reconstruction procedure preoperatively.

In this study, we addressed the question whether 3D-measured RPV has predictive value for POPF in pancreatic cancer patients after PD by comparing various patient-, pancreas-, and surgery-related factors in a retrospective cohort.

\section{Subjects and Methods}

Patients

We retrospectively evaluated 91 consecutive pancreatic cancer patients who underwent PD at Tsukuba Medical Center Hospital, Tsukuba, Japan between January 2007 and December 2016. We used the SYNAPSE VINCENT ${ }^{\circledR}$ medical imaging system (Fujifilm Medical Co., Ltd., Tokyo, Japan) to construct 3D images by integrating MDCT and MRCP images (Fig. 1). This software offers a standardized analysis of liver anatomy and a volumetric risk analysis based on two-dimensional MDCT images [14-16]. In previous studies, we developed 3D reconstructions by integrating MDCT and MRCP images to produce accurate preoperative anatomic images and extended this system to pancreatic surgery [19-22]. RPV was measured using the preoperative 3D image, which simulated actual intraoperative pancreatic parenchymal remnant volume, excluding the 


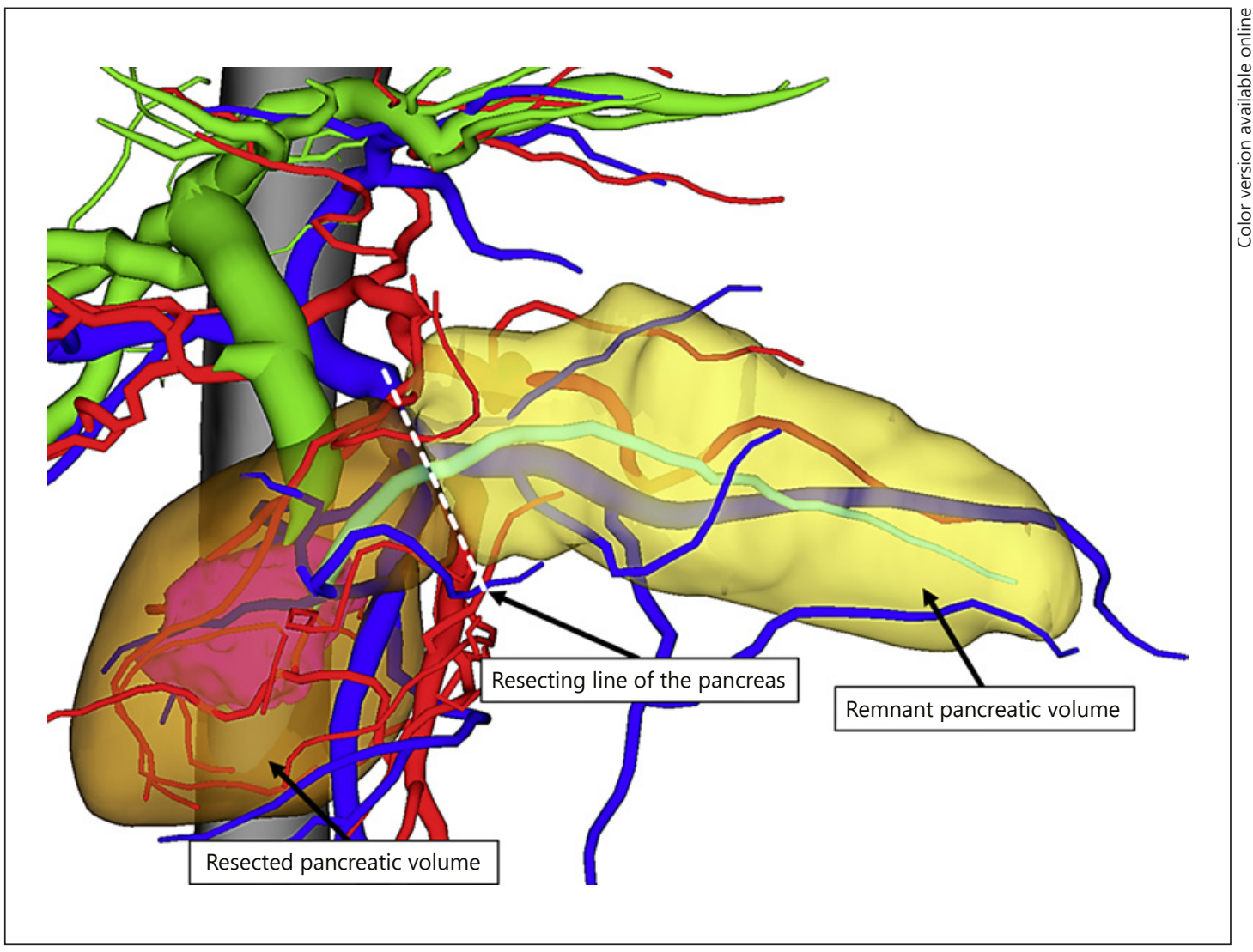

Fig. 1. 3D images of pancreatic tumors obtained by integrating MDCT and MRCP images. This image represents a 3D image view from the front side of the patient. The dashed line represents the resection line of the pancreas. Red represents the arteries, blue the veins, including the portal vein, gray the inferior vena cava, green the biliary duct, turquoise the pancreatic duct, and pink the pancreatic tumor. Light yellow represents the RPV and dark yellow the resected pancreatic volume. 3D, three-dimensional; MDCT, multidetector computed tomography; MRCP, magnetic resonance cholangiopancreatography.

volume of the main pancreatic duct dilation. Each slice of the total pancreatic parenchyma was traced, and the corresponding area was calculated as the sum of the pancreatic tissue areas. Regarding the pancreatic dissection line, we routinely dissected the pancreatic tissue at the left edge of the portal vein. Serial, transverse, enhanced MDCT scan images were obtained at 1.0- or 2.0-mm intervals. The volumetric analysis was conducted by two senior physicians who were blinded to the outcomes.

To examine the discrepancy between preoperative 3D-measured RPV and actual pancreatic volume, we compared preoperative 3D-measured RPV and the pancreatic volume calculated from MDCT scans performed within 1 month after the operation.

We divided the cohort into high- and low-RPV groups based on receiver operating characteristic (ROC) curve analysis cutoff values $\left(>31.5 \mathrm{~cm}^{3}, n=33\right.$, and $\left.\leq 31.5 \mathrm{~cm}^{3}, n=58\right)$. The following patient characteristics were compared between the two groups: age; sex ratio; BMI; American Society of Anesthesiologists score; Eastern Cooperative Oncology Group performance status; preoperative symptoms, including abdominal pain, obstruction, or bleeding; cancer-related biomarkers, including serum carcinoembryonic antigen and carbohydrate antigen 19-9; histological differentiation types; and histopathological stage.

As these factors can also influence perioperative outcomes, including POPF, we collected additional data regarding portal vein resections, pancreatic texture, and main pancreatic duct diameter [23, 24]. We defined pancreatic texture and main pancreatic duct diameter by intraoperative findings or by the pathological status of the pancreatic parenchyma. POPF was defined in accordance with the guidelines of the International Study Group on Pancreatic Fistula [25]. Incidence of POPF was also defined as grade B or C POPF. Furthermore, 
Table 1. Patient characteristics

\begin{tabular}{|c|c|c|c|}
\hline Factor & $\begin{array}{l}\text { Low-RPV group } \\
(n=58)\end{array}$ & $\begin{array}{l}\text { High-RPV group } \\
(n=33)\end{array}$ & $p$ value \\
\hline Age, years & $68(34-86)$ & $69(39-84)$ & 0.31 \\
\hline Sex ratio (male:female) & $32: 26$ & $20: 13$ & 0.14 \\
\hline $\mathrm{BMI}, \mathrm{kg} / \mathrm{m}^{2}$ & $23.7 \pm 2.25$ & $22.8 \pm 2.87$ & 0.54 \\
\hline ASA score (1-4) & $1(1-3)$ & $1(1-3)$ & 0.56 \\
\hline Performance status (0-4) & $0(0-2)$ & $1(0-3)$ & 0.12 \\
\hline History of abdominal surgery & $17(29 \%)$ & $8(24 \%)$ & 0.72 \\
\hline Preoperative symptoms & $35(60 \%)$ & $21(63 \%)$ & 0.22 \\
\hline $\mathrm{CEA}, \mathrm{mg} / \mathrm{dL}$ & $5.58 \pm 3.67$ & $4.98 \pm 4.12$ & 0.15 \\
\hline CA19-9, mg/dL & $101 \pm 394$ & $91.6 \pm 291$ & 0.48 \\
\hline \multicolumn{4}{|l|}{ Stage (UICC 7th edition) } \\
\hline IA & $1(1.7 \%)$ & 0 & \multirow{6}{*}{0.61} \\
\hline IB & $3(5.2 \%)$ & $2(6.1 \%)$ & \\
\hline IIA & $8(1.8 \%)$ & $6(18 \%)$ & \\
\hline IIB & $39(67 \%)$ & $21(64 \%)$ & \\
\hline III & 0 & 0 & \\
\hline IV & $7(12 \%)$ & $4(12 \%)$ & \\
\hline \multicolumn{4}{|l|}{ Histological type } \\
\hline Well & $5(8.6 \%)$ & $2(6.1 \%)$ & \multirow{3}{*}{0.75} \\
\hline Moderately & $48(83 \%)$ & $27(82 \%)$ & \\
\hline Poorly & $5(8.6 \%)$ & $4(12 \%)$ & \\
\hline Portal vein resection & $6(10 \%)$ & $2(6.1 \%)$ & 0.08 \\
\hline Pancreas texture (hard:soft) & $50: 8$ & $15: 18$ & $<0.01^{*}$ \\
\hline Main pancreatic duct diameter, $\mathrm{mm}$ & $5.82 \pm 5.49$ & $2.35 \pm 2.18$ & $<0.01^{*}$ \\
\hline \multicolumn{4}{|c|}{ Postoperative complications (Clavien's classification) } \\
\hline Grade I, II & $60(88 \%)$ & $38(88 \%)$ & \multirow{2}{*}{0.12} \\
\hline Grade III, IV, V & $8(12 \%)$ & $5(12 \%)$ & \\
\hline \multicolumn{4}{|l|}{ Postoperative mortality ( $<90$ days) } \\
\hline Yes & $1(1.5 \%)$ & $1(3.3)$ & \multirow{2}{*}{0.55} \\
\hline No & $67(98 \%)$ & $42(96 \%)$ & \\
\hline Length of postoperative hospital stay, days & $19(8-54)$ & $37(11-62)$ & $0.02^{*}$ \\
\hline
\end{tabular}

ASA, American Society of Anesthesiologists; BMI, body mass index; CA19-9, carbohydrate antigen 19-9; CEA, carcinoembryonic antigen; Moderately, moderately differentiated adenocarcinoma; Poorly, poorly differentiated adenocarcinoma; RPV, remnant pancreatic volume; UICC, Union for International Cancer Control; Well, well-differentiated adenocarcinoma. ${ }^{*} p<0.05$.

when examining perioperative outcomes, we also focused on postoperative complications (Clavien-Dindo classification), excluding the presence of POPF, postoperative mortality ( $<90$ days), and length of postoperative hospital stay.

\section{Patient-, Pancreas-, and Surgery-Related Risk Factors for POPF}

In addition to the 3D-measured RPV value, we also analyzed the relationship between POPF and previously reported POPF risk factors, including patient-, pancreas-, and surgery-related factors, at different treatment phases. Cutoff values for each categorical or continuous variable were defined according to previously reported values or ROC curve analysis [3-7].

Several risk factors for the development of clinically relevant POPF have been defined and subsequently validated, including patient-related risk factors, such as advanced age, male sex, high BMI, preoperative jaundice, chronic kidney disease, and coronary artery diseases; pancreas-related factors, such as pancreatic pathological fibrosis and a small main pancreatic duct $(\leq 3.0 \mathrm{~mm})$; and surgery-related factors, such as long operation time, significant blood loss, less experienced surgeons, pancreaticojejunal anastomotic stents, and pancreaticojejunostomy using the Blumgart or Kakita methods. Less experienced surgeons were defined as surgical residents who were in their fourth to seventh postgraduate year. 
Fig. 2. Correlation between preoperative 3D-measured RPV and postoperative MDCT-measured RPV. 3D, three-dimensional; MDCT, multidetector computed tomography; RPV, remnant pancreatic volume.

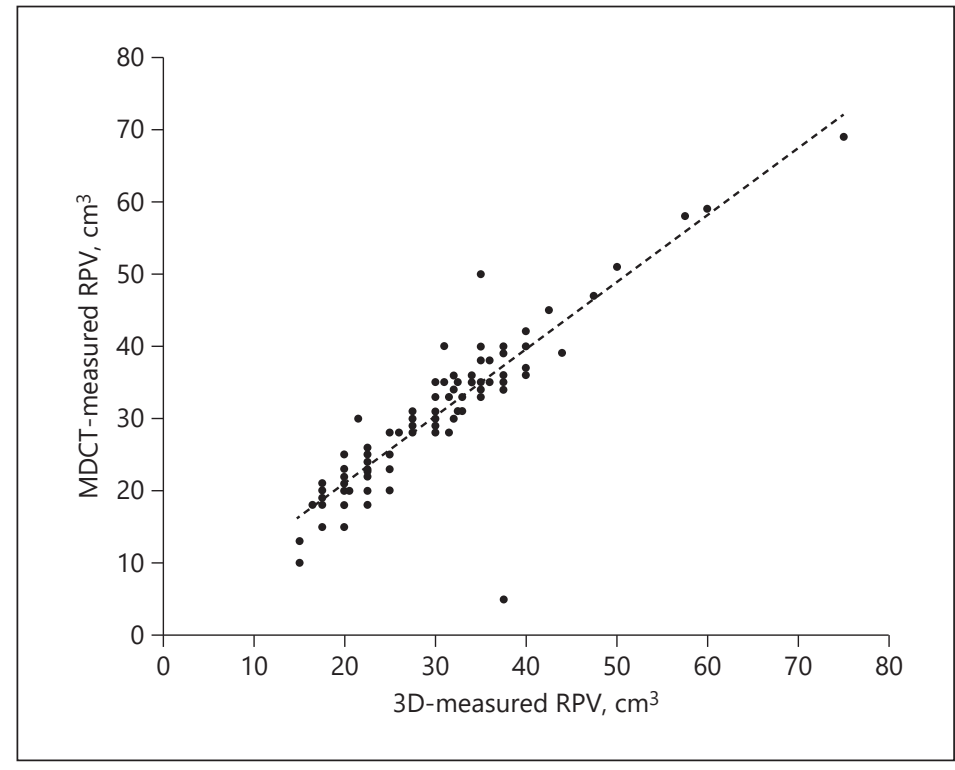

\section{Surgical Procedures}

Ninety-one patients underwent subtotal stomach-preserving PD and modified Child's reconstruction $[26,27]$. Regarding the pancreatic dissection line, we routinely dissected the pancreatic tissue at the left edge of the portal vein. For the pancreaticojejunostomy procedure, we used the Kakita method [28]. From April 2015 onward, we used the Blumgart method to perform pancreaticojejunostomy $[9,10]$. For pancreaticojejunal anastomotic stents, we used internal or external stents depending on the size of the main pancreatic duct [7]. Two drainage tubes were placed superiorly and inferiorly to the anastomotic site between the pancreas and the jejunum. All surgical procedures were performed under the supervision of one or two senior pancreatic surgeons.

\section{Statistical Analyses}

ROC curve analyses were used to evaluate and compare the sensitivity and specificity of the diagnostic tests and to identify cutoff values for continuous variables with positive test results for a certain outcome.

The predictive ability of multiple models based on different factors was described using a concordance index, which is a measure of the predictive ability of a model and is equivalent to the area under the ROC curve. The concordance index ranges from 0.5 to 1.0 , with a value of 0.5 indicating no predictive ability and a value of 1.0 indicating perfect predictive ability. A model is considered reliable when the concordance index is $>0.8$.

Correlations with patient background data were analyzed using the $\chi^{2}$ test or Fisher's exact test, as appropriate. For the multivariate analysis, a multiple logistic regression analysis yielding odds ratios and $95 \%$ confidence intervals was used to identify risk factors for POPF $(p<0.05)$. Statistical analyses were performed using a statistical analysis software package (SPSS Statistics, version 21; IBM, Armonk, NY, USA), and $p$ values $<0.05$ were considered significant.

\section{Results}

\section{Patient Backgrounds}

The backgrounds of the patients, divided into a low- and a high-RPV group, are presented in Table 1. Between the low- and high-RPV groups, significant differences were observed in pancreas texture $(p<0.01)$, main pancreatic duct diameter $(p<0.01)$, and length of postoperative hospital stay $(p=0.02)$. 
Tumors

Table 2. POPF risk factors at different treatment phases

\begin{tabular}{|c|c|c|c|c|c|}
\hline Parameter & $\begin{array}{l}\text { Cutoff } \\
\text { value }\end{array}$ & $\begin{array}{l}\mathrm{Pa}- \\
\text { tients }\end{array}$ & $\begin{array}{l}\text { None and } \\
\text { grade A POPF }\end{array}$ & $\begin{array}{l}\text { Grades B } \\
\text { and C POPF }\end{array}$ & $p$ value \\
\hline \multicolumn{6}{|c|}{ RPV value, $\mathrm{cm}^{3}$} \\
\hline High & \multirow{2}{*}{31.5} & 33 & 15 & 18 & \multirow{2}{*}{$<0.01^{*}$} \\
\hline Low & & 58 & 49 & 9 & \\
\hline \multicolumn{6}{|c|}{ Patient-related risk factors } \\
\hline \multicolumn{6}{|l|}{ Age } \\
\hline High & 70 & 44 & 30 & 14 & \multirow{2}{*}{$0.04^{*}$} \\
\hline Low & 70 & 47 & 38 & 9 & \\
\hline \multicolumn{6}{|l|}{ Sex } \\
\hline Male & & 55 & 40 & 15 & \multirow{2}{*}{0.06} \\
\hline Female & & 36 & 28 & 8 & \\
\hline \multicolumn{6}{|l|}{ BMI, $\mathrm{kg} / \mathrm{m}^{2}$} \\
\hline High & & 49 & 33 & 16 & \multirow{2}{*}{$0.03^{*}$} \\
\hline Low & 25 & 42 & 35 & 7 & \\
\hline \multicolumn{6}{|c|}{ Preoperative jaundice } \\
\hline Yes & & 60 & 47 & 13 & \multirow{2}{*}{0.26} \\
\hline No & & 31 & 21 & 10 & \\
\hline \multicolumn{6}{|c|}{ Chronic kidney disease } \\
\hline Yes & & 15 & 11 & 4 & \multirow{2}{*}{0.51} \\
\hline No & & 76 & 57 & 19 & \\
\hline \multicolumn{6}{|c|}{ Coronary artery disease } \\
\hline Yes & & 21 & 16 & 5 & \multirow{2}{*}{0.31} \\
\hline No & & 70 & 62 & 18 & \\
\hline \multicolumn{6}{|c|}{ Pancreas-related risk factors } \\
\hline \multicolumn{6}{|c|}{ Pathological fibrosis } \\
\hline Yes & & 68 & 57 & 11 & \multirow{2}{*}{$0.01 *$} \\
\hline No & & 23 & 11 & 12 & \\
\hline \multicolumn{6}{|c|}{ Pancreatic duct diameter, $\mathrm{mm}$} \\
\hline High & 30 & 56 & 50 & 6 & \multirow{2}{*}{$<0.01^{*}$} \\
\hline Low & 3.0 & 35 & 18 & 17 & \\
\hline \multicolumn{6}{|c|}{ Surgery-related risk factors } \\
\hline \multicolumn{6}{|c|}{ Operating time, min } \\
\hline High & & 48 & 38 & 10 & \\
\hline Low & 442 & 43 & 30 & 13 & 0.11 \\
\hline Intraoperative & & & & & \\
\hline High & & 47 & 34 & 13 & \\
\hline Low & 538 & 44 & 34 & 10 & 0.48 \\
\hline Less experien & & & & & \\
\hline Yes & & 26 & 17 & 9 & 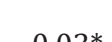 \\
\hline No & & 65 & 51 & 14 & $0.03^{*}$ \\
\hline Internal drain & & & & & \\
\hline Yes & & 65 & 50 & 15 & ר? \\
\hline No & & 26 & 18 & 8 & 0.33 \\
\hline Type of pancr & & & & & \\
\hline Kakita & & 73 & 48 & 25 & $001 *$ \\
\hline Blumgart & & 18 & 15 & 3 & $<0.01^{*}$ \\
\hline
\end{tabular}

BMI, body mass index; POPF, postoperative pancreatic fistula; RPV, remnant pancreatic volume. ${ }^{*} p<0.05$. 
Table 3. Multivariate analysis of POPF risk factors

\begin{tabular}{lccc}
\hline Parameter & HR & $95 \%$ CI & $p$ value \\
\hline High RPV $\left(>31.5 \mathrm{~cm}^{3}\right)$ & 8.41 & $3.27-16.1$ & $0.01^{*}$ \\
Age $(>70$ years) & 0.53 & $0.24-1.23$ & 0.12 \\
BMI $\left(>25 \mathrm{~kg} / \mathrm{m}^{2}\right)$ & 1.53 & $0.42-1.59$ & $0.02^{*}$ \\
No pathological fibrosis & 3.41 & $2.20-8.22$ & $<0.01^{*}$ \\
Pancreatic duct diameter $(\leq 3.0 \mathrm{~mm})$ & 5.48 & $3.12-12.6$ & $<0.01^{*}$ \\
Type of pancreaticojejunal anastomosis (Kakita method) & 0.73 & $0.35-1.41$ & 0.31 \\
Less experienced surgeon & 0.99 & $0.61-1.68$ & 0.83 \\
\hline
\end{tabular}

BMI, body mass index; CI, confidence interval; HR, hazard ratio; POPF, postoperative pancreatic fistula; $\mathrm{RPV}$, remnant pancreatic volume. ${ }^{*} p<0.05$.

\section{Correlation between Preoperative 3D-Measured RPV and Postoperative MDCT-Measured $R P V$}

A significant correlation was observed between preoperative 3D-measured RPV and pancreatic volume calculated from MDCT scans performed within 1 month after the operation $(r=0.914, p=0.02$ ) (Fig. 2).

Univariate Analyses for POPF Risk Factors at Different Treatment Phases (Table 2)

A significant difference in the RPV value was observed with respect to the incidence of POPF (high: 18 [55\%]; low: 9 [16\%]; $p<0.01$ ). In terms of patient-related risk factors, age (high: 14 [32\%]; low: 9 [19\%]; $p=0.04$ ) and BMI (high: 16 [33\%]; low: 7 [17\%]; $p=0.03$ ) showed significant differences with respect to the incidence of POPF. In terms of pancreasrelated risk factors, pathological fibrosis (yes: 14 [16\%]; no: 12 [52\%]; $p=0.01$ ) and pancreatic duct diameter (>3.0 mm: 6 [11\%]; $\leq 3.0 \mathrm{~mm}: 17$ [49\%]; $p<0.01$ ) showed significant differences with respect to the incidence of POPF. In terms of surgery-related risk factors, less experienced surgeons (yes: 9 [35\%]; no: 14 [22\%]; $p=0.03$ ) and the type of pancreaticojejunal anastomosis (Kakita: 30 [34\%]; Blumgart: 3 [17\%]; $p<0.01$ ) showed significant differences with respect to the incidence of POPF.

Multivariate Analysis (Table 3)

Multivariate logistic regression analysis also identified a high RPV value $\left(>31.5 \mathrm{~cm}^{3}\right)$ (hazard ratio $[\mathrm{HR}]=8.41, p=0.01)$, pancreatic duct diameter $\leq 3.0 \mathrm{~mm}(\mathrm{HR}=5.48, p<0.01)$, no pathological fibrosis (HR $=3.41, p<0.01)$, and BMI $>25 \mathrm{~kg} / \mathrm{m}^{2}(\mathrm{HR}=1.53, p=0.02)$ as independent risk factors for POPF. The sensitivity, specificity, positive predictive value, and negative predictive value of an RPV value $>31.5 \mathrm{~cm}^{3}$, pancreatic duct diameter $\leq 3.0 \mathrm{~mm}$, lack of pathological fibrosis, and BMI $>25 \mathrm{~kg} / \mathrm{m}^{2}$ are shown in Table 4 .

\section{Discussion}

In the present study, we found that preoperative 3D-measured RPV was a risk factor for POPF, as were pancreatic duct diameter, no pathological fibrosis, and increased BMI. Therefore, we assumed that preoperative 3D-measured RPV has additional useful predictive value for POPF in pancreatic cancer patients after PD.

Our multivariate analysis indicated that a 3D-measured RPV value $>31.5 \mathrm{~cm}^{3}$ was the strongest risk factor for POPF (HR $=8.41$; Table 3 ). Since the RPV value is objectively 
Miyamoto et al.: Three-Dimensional-Measured Pancreatic Volumetry

Table 4. Comparison of diagnostic outcomes among four predictive values for POPF

\begin{tabular}{lllll}
\hline Parameter & $\begin{array}{l}\text { Sensitivity, } \% \\
(95 \% \mathrm{CI})\end{array}$ & $\begin{array}{l}\text { Specificity, } \% \\
(95 \% \mathrm{CI})\end{array}$ & $\begin{array}{l}\text { PPV, } \% \\
(95 \% \mathrm{CI})\end{array}$ & $\begin{array}{l}\text { NPV, \% } \\
(95 \% \mathrm{CI})\end{array}$ \\
\hline $\begin{array}{l}\text { RPV }>31.5 \mathrm{~cm}^{3} \\
\text { Pancreatic duct diameter }\end{array}$ & $75.3(0.632-0.814)$ & $72.9(0.632-0.801)$ & $81.5(0.711-0.865)$ & $82.5(0.735-0.896)$ \\
$\quad$ <3.0 mm & $78.8(0.720-0.816)$ & $81.8(0.760-0.856)$ & $78.5(0.642-0.825)$ & $81.9(0.605-0.858)$ \\
No pathological fibrosis & $67.5(0.689-0.875)$ & $78.8(0.750-0.803)$ & $72.5(0.642-0.825)$ & $71.9(0.605-0.778)$ \\
BMI $>25 \mathrm{~kg} / \mathrm{m}^{2}$ & $69.7(0.547-0.771)$ & $67.2(0.689-0.897)$ & $71.5(0.641-0.798)$ & $65.3(0.554-0.740)$ \\
\hline
\end{tabular}

BMI, body mass index; CI, confidence interval; NPV, negative predictive value; POPF, postoperative pancreatic fistula; PPV, positive predictive value; RPV, remnant pancreatic volume.

measured by preoperative 3D images, we conclude that, compared with other well-known risk factors such as pancreatic duct diameter and pancreatic texture, RPV is more useful for predicting clinically relevant POPF. The early identification of patients at a high risk for clinically relevant POPF can increase the accuracy of preoperative benefit/risk assessment and the information given to patients. Furthermore, early identification should be useful for guiding technical and pharmacologic management practices, including perioperative nutritional support, imaging examinations for the early detection of abdominal abscesses, infection control, drainage tube management, and the administration of somatostatin analogs [11-13].

A potential correlation between RPV values and the presence of POPF has been well discussed $[13,17,18]$. Frozanpor et al. [18] and Kirihara et al. [13] reported that RPV values calculated using preoperative MDCT images were a useful predictor of POPF; however, patients with pancreatic head cancer often have atrophic changes in the pancreatic parenchyma and subsequent main pancreatic duct dilation, leading to an overestimation of RPV. Moreover, Kanda et al. [17] reported that RPV, excluding the remnant volume of the main pancreatic duct, was a better predictor of clinically relevant POPF. However, the RPV values estimated from MDCT images alone are fairly imprecise. First, these methods for pancreatic remnant volumetry are insufficient to simulate the actual pancreatic dissection position. By contrast, our 3D technique enables us to simulate 3D anatomic images to enhance the actual pancreatic dissection position. Second, 3D images allow for the precise calculation of organ volumetry. 3D-measured volumetry is widely used for hepatic resection to facilitate surgical planning and to determine the future liver remnant volume using preoperative imaging, allowing one to predict which patients are at high risk of hepatic failure after liver resection [14-16]. Such patients are selected for portal vein embolization to promote hypertrophy of the future liver remnant and decrease postoperative morbidity. In a previous study, we showed that 3D imaging is useful for understanding and sharing anatomic information, particularly concerning the main pancreatic duct, in pancreatic surgery [19-22]. Furthermore, the present study found no significant differences between preoperative 3D-measured RPV and postoperative MDCT-measured pancreatic volume. Therefore, we proposed that the application of this 3D imaging technique would enable us to precisely estimate the RPV, excluding the volume of the main pancreatic duct dilation.

This novel 3D imaging technique has several difficulties that must be addressed. First, a considerable amount of time (approximately $3-5 \mathrm{~h}$ at present) is required to create the detailed 3D images because of the performance of the software system. Second, the internal organs of the abdomen are affected by respiratory motion, and the degree of movement varies with each phase. To obtain a more precise and accurate preoperative 3D image, we typically 
used anatomic landmarks, i.e., the junction between the portal and splenic veins and the bifurcation of the gastroduodenal and hepatic arteries [19].

There remain significant questions regarding the mechanisms by which high RPV values contribute to the presence of POPF. RPV is associated with pancreatic fluid volume and drainage fluid amylase activity, consistent with the hypothesis that patients with a greater pancreatic parenchymal remnant volume have a greater volume of pancreatic fluid secretion, resulting in a greater rate of clinically relevant POPF [12]. In addition, increased RPV values showed a positive correlation with a soft pancreatic texture and a small main pancreatic duct size $(\leq 3.0 \mathrm{~mm})[7,8,29]$. Indeed, in the present study, we also found significant differences in pancreas texture $(p<0.01)$ and main pancreatic duct diameter $(p<0.01)$ between the lowand high-RPV groups. With respect to the pancreaticojejunostomy procedure, a soft pancreatic parenchyma and a small main pancreatic duct $(\leq 3.0 \mathrm{~mm})$ can make anastomosis more technically challenging, leading to surgery-related POPF. In fact, our multivariate analysis indicated that pancreatic duct diameter $\leq 3.0 \mathrm{~mm}$ and soft pancreatic texture without pathological fibrosis are independent risk factors for POPF.

In conclusion, high RPV values were associated with a significantly increased risk of clinically relevant POPF. These measurements were more sensitive than the classic parameters of pancreatic duct size, parenchymal texture, and BMI. Our novel modeling technique, which is based on 3D imaging, appears sufficient to classify patients into high- and low-RPV groups. We conclude that preoperative 3D-measured RPV has useful predictive value for POPF in pancreatic cancer patients after PD. The present study had limitations, including the fact that this was a retrospective single-center study with a relatively small patient cohort. These results require confirmation by additional multicenter large-scale studies.

\section{Statement of Ethics}

The ethics committee of Tsukuba Medical Center Hospital approved this study (\#2018-010).

\section{Disclosure Statement}

The authors have no conflicts of interest or financial ties to disclose.

\section{References}

1 Nakao A, Kanzaki A, Fujii T, Kodera Y, Yamada S, Sugimoto H, et al. Correlation between radiographic classification and pathological grade of portal vein wall invasion in pancreatic head cancer. Ann Surg. 2012 Jan; 255(1):103-8.

2 Farnell MB, Pearson RK, Sarr MG, DiMagno EP, Burgart LJ, Dahl TR, et al.; Pancreas Cancer Working Group. A prospective randomized trial comparing standard pancreatoduodenectomy with pancreatoduodenectomy with extended lymphadenectomy in resectable pancreatic head adenocarcinoma. Surgery. 2005 Oct;138(4): 618-28.

3 Ahmad SA, Edwards MJ, Sutton JM, Grewal SS, Hanseman DJ, Maithel SK, et al. Factors influencing readmission after pancreaticoduodenectomy: a multi-institutional study of 1302 patients. Ann Surg. 2012 Sep;256(3): 529-37.

4 McMillan MT, Soi S, Asbun HJ, Ball CG, Bassi C, Beane JD, et al. Risk-adjusted Outcomes of Clinically Relevant Pancreatic Fistula Following Pancreatoduodenectomy: A Model for Performance Evaluation. Ann Surg. 2016 Aug;264(2):344-52.

5 Miller BC, Christein JD, Behrman SW, Drebin JA, Pratt WB, Callery MP, et al. A multi-institutional external validation of the fistula risk score for pancreatoduodenectomy. J Gastrointest Surg. 2014 Jan;18(1):172-9.

6 Gaujoux S, Cortes A, Couvelard A, Noullet S, Clavel L, Rebours V, et al. Fatty pancreas and increased body mass index are risk factors of pancreatic fistula after pancreaticoduodenectomy. Surgery. 2010 Jul;148(1):15-23. 
7 Poon RT, Fan ST, Lo CM, Ng KK, Yuen WK, Yeung C, et al. External drainage of pancreatic duct with a stent to reduce leakage rate of pancreaticojejunostomy after pancreaticoduodenectomy: a prospective randomized trial. Ann Surg. 2007 Sep;246(3):425-33.

$8 \mathrm{Hu}$ BY, Wan T, Zhang WZ, Dong JH. Risk factors for postoperative pancreatic fistula: analysis of 539 successive cases of pancreaticoduodenectomy. World J Gastroenterol. 2016 Sep;22(34):7797-805.

9 Kimura W, Miyata H, Gotoh M, Hirai I, Kenjo A, Kitagawa Y, et al. A pancreaticoduodenectomy risk model derived from 8575 cases from a national single-race population (Japanese) using a web-based data entry system: the 30-day and in-hospital mortality rates for pancreaticoduodenectomy. Ann Surg. 2014 Apr;259(4): 773-80.

10 Kleespies A, Rentsch M, Seeliger H, Albertsmeier M, Jauch KW, Bruns CJ. Blumgart anastomosis for pancreaticojejunostomy minimizes severe complications after pancreatic head resection. Br J Surg. 2009 Jul;96(7): 741-50.

11 Oda T, Hashimoto S, Miyamoto R, Shimomura O, Fukunaga K, Kohno K, et al. The tight adaptation at pancreatic anastomosis without parenchymal laceration: an institutional experience in introducing and modifying the new procedure. World J Surg. 2015 Aug;39(8):2014-22.

12 Muscari F, Suc B, Kirzin S, Hay JM, Fourtanier G, Fingerhut A, et al.; French Associations for Surgical Research. Risk factors for mortality and intra-abdominal complications after pancreatoduodenectomy: multivariate analysis in 300 patients. Surgery. 2006 May;139(5):591-8.

13 Kirihara Y, Takahashi N, Hashimoto Y, Sclabas GM, Khan S, Moriya T, et al. Prediction of pancreatic anastomotic failure after pancreatoduodenectomy: the use of preoperative, quantitative computed tomography to measure remnant pancreatic volume and body composition. Ann Surg. 2013 Mar;257(3):512-9.

14 Mise Y, Tani K, Aoki T, Sakamoto Y, Hasegawa K, Sugawara Y, et al. Virtual liver resection: computer-assisted operation planning using a three-dimensional liver representation. J Hepatobiliary Pancreat Sci. 2013 Feb; 20(2):157-64.

15 Nakayama K, Oshiro Y, Miyamoto R, Kohno K, Fukunaga K, Ohkohchi N. The Effect of Three-Dimensional Preoperative Simulation on Liver Surgery. World J Surg. 2017 Jul;41(7):1840-7.

16 Takahashi K, Sasaki R, Kondo T, Oda T, Murata S, Ohkohchi N. Preoperative 3D volumetric analysis for liver congestion applied in a patient with hilar cholangiocarcinoma. Langenbecks Arch Surg. 2010 Aug;395(6): 761-5.

17 Kanda M, Fujii T, Suenaga M, Takami H, Hattori M, Inokawa Y, et al. Estimated pancreatic parenchymal remnant volume accurately predicts clinically relevant pancreatic fistula after pancreatoduodenectomy. Surgery. 2014 Sep;156(3):601-10.

18 Frozanpor F, Loizou L, Ansorge C, Lundell L, Albiin N, Segersvärd R. Correlation between preoperative imaging and intraoperative risk assessment in the prediction of postoperative pancreatic fistula following pancreatoduodenectomy. World J Surg. 2014 Sep;38(9):2422-9.

19 Oshiro Y, Sasaki R, Nasu K, Ohkohchi N. A novel preoperative fusion analysis using three-dimensional MDCT combined with three-dimensional MRI for patients with hilar cholangiocarcinoma. Clin Imaging. 2013 JulAug;37(4):772-4.

20 Miyamoto R, Oshiro Y, Hashimoto S, Kohno K, Fukunaga K, Oda T, et al. Three-dimensional imaging identified the accessory bile duct in a patient with cholangiocarcinoma. World J Gastroenterol. 2014 Aug;20(32): $11451-5$.

21 Miyamoto R, Oshiro Y, Nakayama K, Kohno K, Hashimoto S, Fukunaga K, et al. Three-dimensional simulation of pancreatic surgery showing the size and location of the main pancreatic duct. Surg Today. 2017 Mar;47(3): 357-64.

22 Miyamoto R, Oshiro Y, Nakayama K, Ohkohchi N. Impact of Three-Dimensional Surgical Simulation on Pancreatic Surgery. Gastrointest Tumors. 2018 Feb;4(3-4):84-9.

23 Fuks D, Piessen G, Huet E, Tavernier M, Zerbib P, Michot F, et al. Life-threatening postoperative pancreatic fistula (grade C) after pancreaticoduodenectomy: incidence, prognosis, and risk factors. Am J Surg. 2009 Jun; 197(6):702-9.

24 Akamatsu N, Sugawara Y, Komagome M, Shin N, Cho N, Ishida T, et al. Risk factors for postoperative pancreatic fistula after pancreaticoduodenectomy: the significance of the ratio of the main pancreatic duct to the pancreas body as a predictor of leakage. J Hepatobiliary Pancreat Sci. 2010 May;17(3):322-8.

25 Bassi C, Dervenis C, Butturini G, Fingerhut A, Yeo C, Izbicki J, et al.; International Study Group on Pancreatic Fistula Definition. Postoperative pancreatic fistula: an international study group (ISGPF) definition. Surgery. 2005 Jul;138(1):8-13.

26 Matsumoto I, Shinzeki M, Asari S, Goto T, Shirakawa S, Ajiki T, et al. A prospective randomized comparison between pylorus- and subtotal stomach-preserving pancreatoduodenectomy on postoperative delayed gastric emptying occurrence and long-term nutritional status. J Surg Oncol. 2014 Jun;109(7):690-6.

27 Hayashibe A, Kameyama M, Shinbo M, Makimoto S. The surgical procedure and clinical results of subtotal stomach preserving pancreaticoduodenectomy (SSPPD) in comparison with pylorus preserving pancreaticoduodenectomy (PPPD). J Surg Oncol. 2007 Feb;95(2):106-9.

28 Kakita A, Yoshida M, Takahashi T. History of pancreaticojejunostomy in pancreaticoduodenectomy: development of a more reliable anastomosis technique. J Hepatobiliary Pancreat Surg. 2001;8(3):230-7.

29 Callery MP, Pratt WB, Kent TS, Chaikof EL, Vollmer CM Jr. A prospectively validated clinical risk score accurately predicts pancreatic fistula after pancreatoduodenectomy. J Am Coll Surg. 2013 Jan;216(1):1-14. 